\title{
Performance Improvement of Collision Warning System on Curved Road Based on Intervehicle Communication
}

\author{
Hong Cho ${ }^{1}$ and Byeong-woo $\mathrm{Kim}^{2}$ \\ ${ }^{1}$ Graduate School of Electrical Engineering, University of Ulsan, 93 Daehak-ro, Ulsan 680-749, Republic of Korea \\ ${ }^{2}$ School of Electrical Engineering, University of Ulsan, 93 Daehak-ro, Ulsan 680-749, Republic of Korea
}

Correspondence should be addressed to Byeong-woo Kim; bywokim@ulsan.ac.kr

Received 20 September 2014; Accepted 15 February 2015

Academic Editor: Jong-Hyuk Park

Copyright (C) 2015 H. Cho and B.-w. Kim. This is an open access article distributed under the Creative Commons Attribution License, which permits unrestricted use, distribution, and reproduction in any medium, provided the original work is properly cited.

\begin{abstract}
The vehicle on-board sensor based Advanced Driver Assistant System possesses limitations on a small road with a small radius of curvature because of the sensor's inability to operate in nondetectable domains. This study suggests an Improved Cooperative Collision Warning System (ICCWS) that considers the curvature of the road and is based on intervehicle communication. To predict the radius of curvature of the road, the Arc Relative Distance (ARD), the real relative distance to a preceding vehicle on a curved road has been used. The risk of collision with the preceding vehicle is decided by calculating an index of the risk of collision on a curved road using the computed ARD. The effects of ICCWS, proposed through this simulation, have been reviewed, and the improvement in performance in following a preceding vehicle has been analyzed quantitatively via comparative analysis with the conventional forward collision warning system. Accordingly, if the estimating algorithm for curvature developed in this study is applied to a real system, the performance of following a preceding vehicle can be improved without any specific changes to the system.
\end{abstract}

\section{Introduction}

The number of road-traffic accidents is rising proportionally in line with an increase in the number of vehicles on the road. Based on data reported by the National Highway Traffic Safety Administration (NHTSA) [1], about $80 \%$ of traffic accidents are due to the carelessness of drivers. Recently, studies related to an Advanced Driver Assistant System (ADAS) have actively been carried out, not only to reinforce legal regulations of vehicle safety, but also to increase awareness of safety devices available to consumers and decrease the possibility of the traffic accidents related to driver carelessness. The ADAS is a representative vehicle safety system that detects stopping or moving objects using sensors such as camera and radar and classifies them to reduce the possibility of an accident [2]. The major longitudinal ADAS is composed of a forward collision warning system (FCWS) and an Adaptive Cruise Control System (ACCS). The FCWS is a system that analyzes the risk of collision with a preceding vehicle and operates by using a vehicle on-board sensor to warn the driver of a collision. However, the vehicle on-board sensor based on ADAS only works within a measurable range of the sensor and possesses blind spots in relation to areas that it is unable to detect, such as at crossroads and on curved roads. In order to overcome these limitations, studies introducing a communication-connected safety system using Vehicle to Vehicle (V2V) communication and Vehicle to Infra (V2I) communication have actively been planned in relation to further developments made in communication technology [3-5].

The FCWS has already been established as an international standard by the International Organization for Standardization (ISO) [6]. However, the existing FCWS uses vehicle-mounted sensors that cause a malfunctioning of the system when the object in front enters a blind spot, thus escaping the measurable range of the sensor [7]. In order to overcome the limitations related to such blind spots, studies on the Cooperative Collision Warning System (CCWS) have been carried out by combining V2V and V2I technology [8]. However, the CCWS proposed thus far is not suitable for 


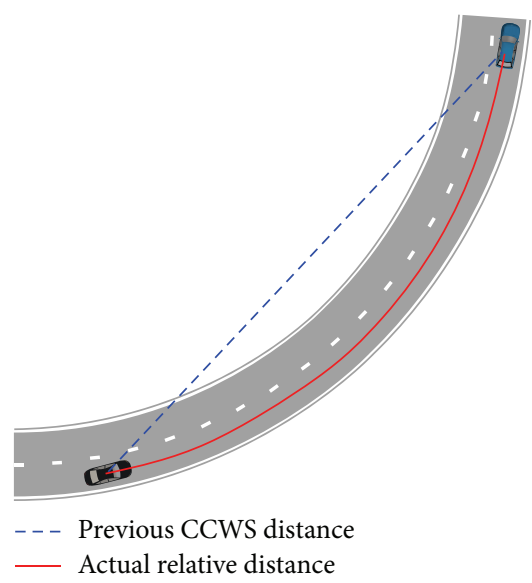

FIGURE 1: Limitations of CCWS.

narrow curved roads with a small radius of curvature, and it can only consider straight roads and slightly curved narrow roads. It is therefore considered that, to overcome such limitations, the development of a collision warning system that considers the curvature of curved roads is required. Thus, this study proposes an Improved Cooperative Collision Warning System (ICCWS) to overcome the problems of the conventional FCWS and CCWS without adding devices to a vehicle.

\section{Improved Cooperative Collision Warning System Design}

CCWSs proposed in previous studies use V2V communication to overcome the limitations of vehicle-mounted sensors and to warn the driver in advance by detecting the risk of collision with preceding vehicles, regardless of the existence of obstacles [9]. However, as shown in Figure 1, CCWSs proposed in previous studies are not suitable for curved roads with a small radius of curvature. Therefore, this paper proposes an ICCWS, which takes into account the curvature of the road. Figure 2 shows a block diagram of the ICCWS proposed in this study, which firstly calculates the relative distance $(\mathrm{RD})$ and relative angle (RA) between nearby vehicles using the vehicle surroundings monitoring system according to whether the road is curved or straight and then applies the ICCWS to an ego-vehicle.

2.1. Vehicle Surroundings Monitoring System. As shown in Figure 3, the system generates coordinates based on the egovehicle and calculates the RA and RD to any surrounding vehicles. In this study, a new Cartesian coordinate system, the $\mathrm{CS}_{\text {ego }}$, was defined using the ego-vehicle's current position $\left(x_{\text {ego }}, y_{\text {ego }}\right)$, as the starting point. The $\mathrm{CS}_{\text {ego }}$ defined here is a coordinate system in which the longitudinal direction is the $x$-axis and the lateral direction is the $y$-axis, based on the traveling direction of the ego-vehicle. In the $\mathrm{CS}_{\text {ego }}$, the positions of the surrounding vehicles are indicated as relative coordinates $\left(x_{n}, y_{n}\right)$ in the four quadrants of the $\mathrm{CS}_{\text {ego }}$ by comparing the positions of the ego-vehicle and the surrounding vehicles that are received through $\mathrm{V} 2 \mathrm{~V}$ communication.
Here, $n$ is vehicle ID. $\mathrm{RA}_{n}$ is then calculated using a comparison with the azimuth, $\varphi$, which is related to the traveling direction of the ego-vehicle. As shown in Figure 3, based on $\mathrm{RA}_{n}$, which is the relative angle that changes according to the four quadrants, the vehicle surroundings monitoring system can detect the position of surrounding vehicles. As shown in Figure 4, $\varphi$ changes according to the heading angle of the vehicle, and East is designated as $0^{\circ}$. The $\mathrm{CS}_{\text {ego }}$, as shown in Figure 4, rotates its axes depending on any changes made to the vehicle's $\varphi$. Considering this axis rotation, the longitudinal vehicle travelling direction was set to align with the $x$-axis at all times and is represented by

$$
\left[\begin{array}{l}
x^{\prime} \\
y^{\prime}
\end{array}\right]=\left[\begin{array}{cc}
\cos \varphi & \sin \varphi \\
-\sin \varphi & \cos \varphi
\end{array}\right]\left[\begin{array}{l}
x \\
y
\end{array}\right] .
$$

2.2. Curved Road Collision Detection System. In this study, the time to collision (TTC) was used as the collision risk index to determine the risk of a collision with a preceding vehicle. TTC refers to the time remaining before impact, which is determined by the ratio of the distance to the preceding vehicle and the relative speed, in accordance with

$$
\text { TTC }(s)=\frac{\text { Relative distance }}{\text { Relative speed }} \text {. }
$$

Under curved road conditions, $\mathrm{RD}$, which is the relative distance between the ego-vehicle and the forward vehicle calculated by the vehicle surroundings monitoring system, is not suitable for calculating TTC, the longitudinal collision risk index. Therefore, the actual relative distance needs to be defined by taking the conditions of the curved road into consideration. In this study, as shown in Figure 5, the RD and turning radius $\left(\mathrm{TR}_{\text {ego }}\right)$ of the ego-vehicle were used to calculate the actual relative distance (the Arc Relative Distance (ARD)) for a curved road, as defined using the following:

$$
\mathrm{ARD}_{n}=\frac{\theta_{n}}{180} \cdot \pi \cdot \mathrm{TR}_{\text {ego }}
$$

where $\mathrm{ARD}_{n}$ is the relative distance from the forward vehicle, $n$, on a curved road; $\mathrm{TR}_{\text {ego }}$ is the ego-vehicle's turning radius; $\theta_{n}$ is the mid angle when the ego-vehicle's turning radius is $\mathrm{TR}_{\text {ego }}$ and the ego-vehicle's preceding vehicle, $n$, is distanced by $\mathrm{ARD}_{n}$. The vehicle's TR can be determined through

$$
\mathrm{TR}_{n}=\frac{V_{n}}{\dot{\psi}},
$$

where $V_{n}$ is the speed of the vehicle and $\dot{\psi}_{n}$ is the yaw rate of the vehicle.

The ICCWS can only operate on preceding vehicles in the same lane. For this, when the distance between $\mathrm{TR}_{\text {ego }}$ and $\mathrm{TR}_{n}$ is less than half the width of the lane, it was determined that the ego-vehicle and the preceding vehicle, $n$, are in the same lane. When $\mathrm{TR}_{n}$ could not be determined because the preceding vehicle, $n$, had stopped, it was estimated through the following (Figure 6) [10]:

$$
\mathrm{TR}_{n}=\sqrt{\left(\mathrm{TR}_{\mathrm{ego}}-\mathrm{RD}_{n} \cdot \sin \left(\mathrm{RA}_{n}\right)\right)^{2}-\left(\mathrm{RD}_{n} \cdot \cos \left(\mathrm{RA}_{n}\right)\right)^{2}} .
$$




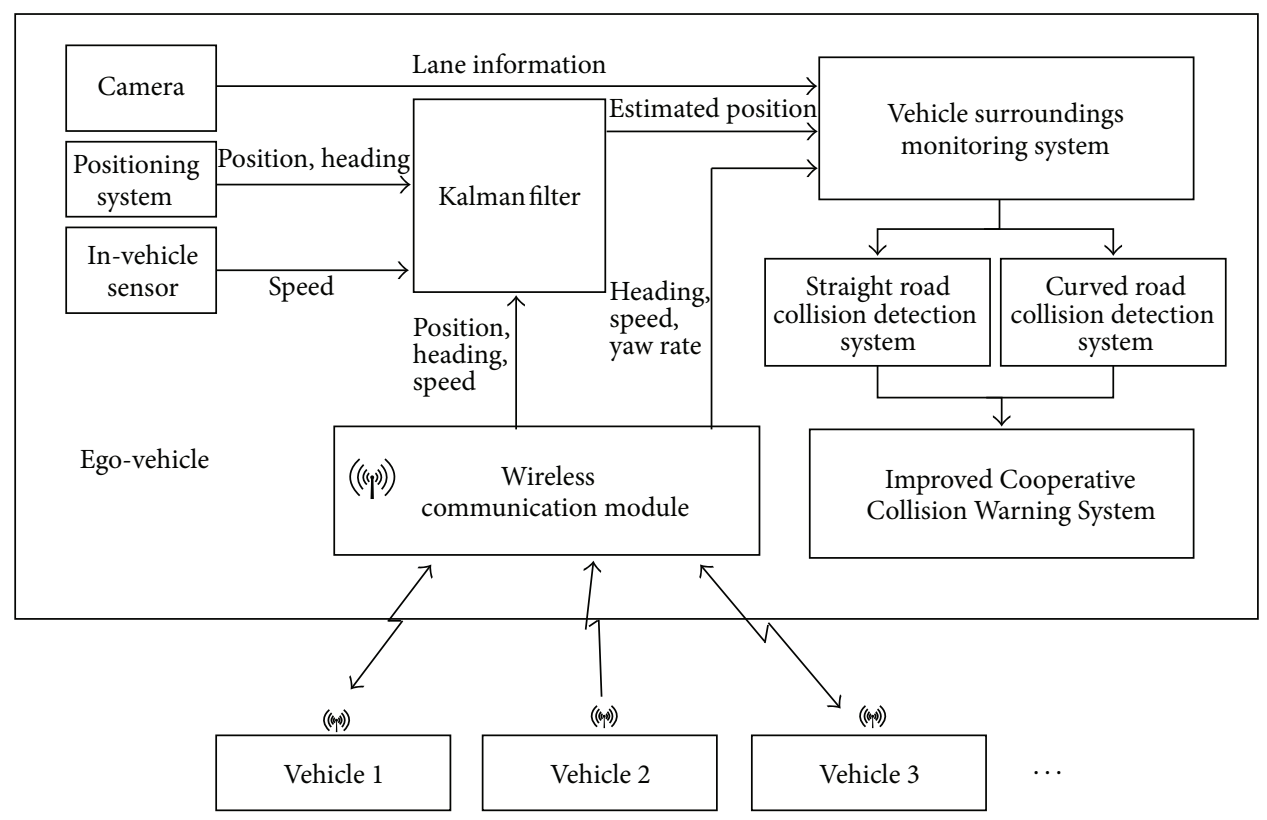

FIGURE 2: Block diagram for ICCWS.

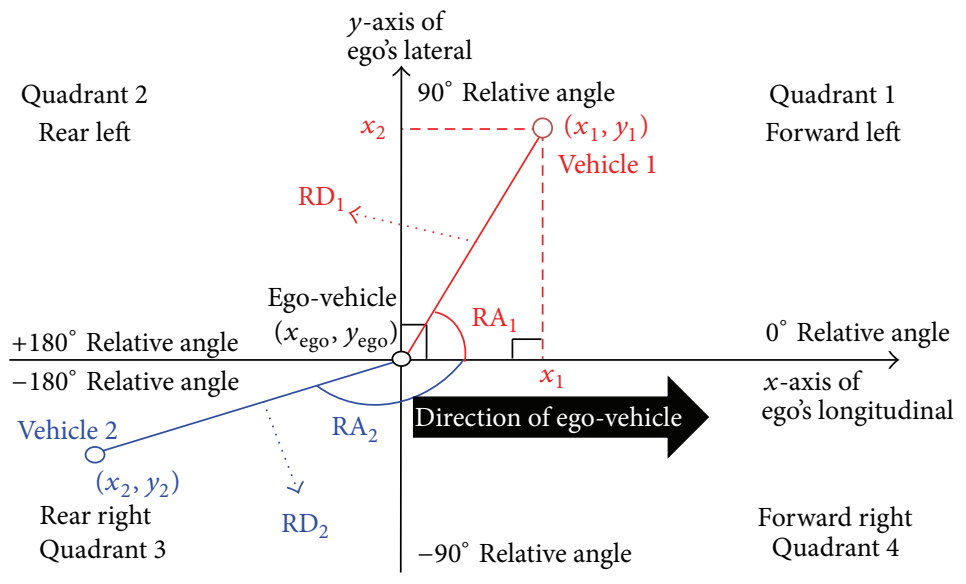

FIgURE 3: Principles of the vehicle surroundings monitoring system.

\section{Simulation and Results}

Commercial programs such as PreScan, CarSim, and MAT$\mathrm{LAB} /$ Simulink were used to conduct the simulation. PreScan was used to perform the following modeling operations: (1) configuration of the V2V communication environment; (2) vehicle-mounted camera and radar sensor modeling; (3) modeling of vehicle surroundings; and (4) modeling in relation to driving conditions. Furthermore, a CarSim vehicle model was used for expressing the detailed kinetic characteristics of the ego-vehicle. Finally, the system was configured after interfacing the PreScan and CarSim through the MATLAB/Simulink.

3.1. Simulation Scenario. The simulation scenario is shown in Figure 7. The ICCWS warning times for an ego-vehicle travelling on a curved road were observed for a case when
Vehicle 1 was travelling ahead of the ego-vehicle. In this context, the initial and driving speeds of the ego-vehicle were standard speeds corresponding to the curvature radius of the curved road with a superelevation of $6 \%$, but in the established scenario the driving speed was exceeded by $5 \mathrm{~km} / \mathrm{h}$ and $10 \mathrm{~km} / \mathrm{h}$, respectively. Table 1 presents the standard vehicle speeds on the curved road corresponding to various curvature radii, with a superelevation of $6 \%$. The warning levels were defined based on the TTC; Table 2 lists the warning level standards corresponding to variations in the TTC [7]. The warning level was divided into a total of three levels: Level 1 was defined as the lowest level of risk, Level 2 as intermediate risk, and Level 3 as the highest risk.

3.2. Simulation Results. The proposed ICCWS was verified by comparing the ideal $\mathrm{TTC}_{x}$ with the $\mathrm{TTC}_{x}$ calculated from the ICCWS. The ideal $\mathrm{TTC}_{x}$ can be calculated using the 


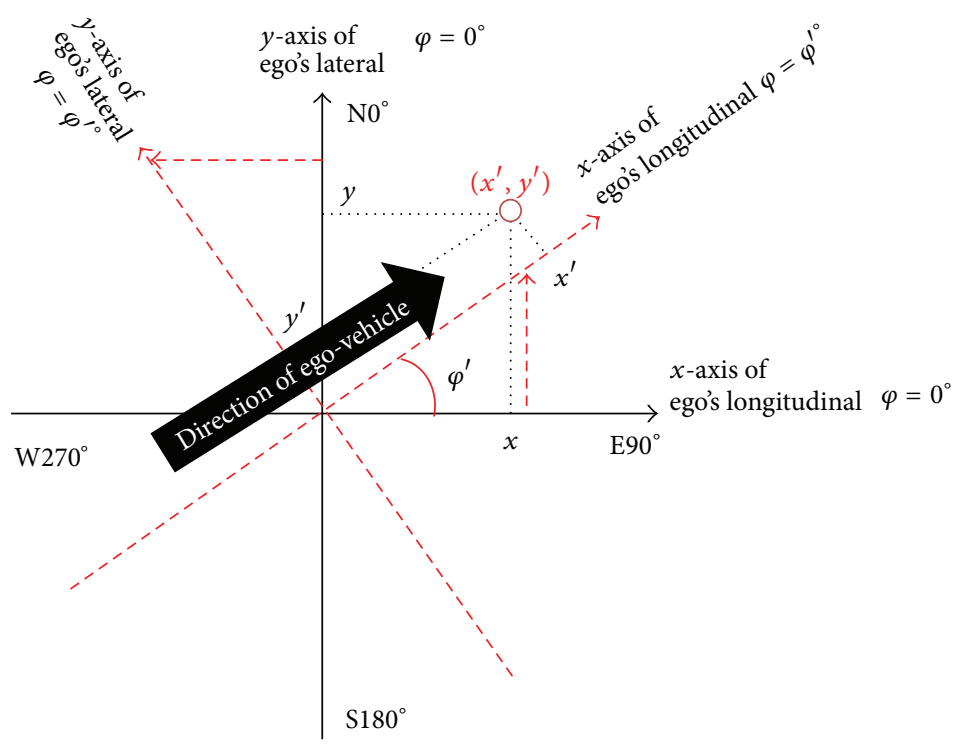

FIGURE 4: Rotations of $\mathrm{CS}_{\text {ego }}$ due to changes in the azimuth.

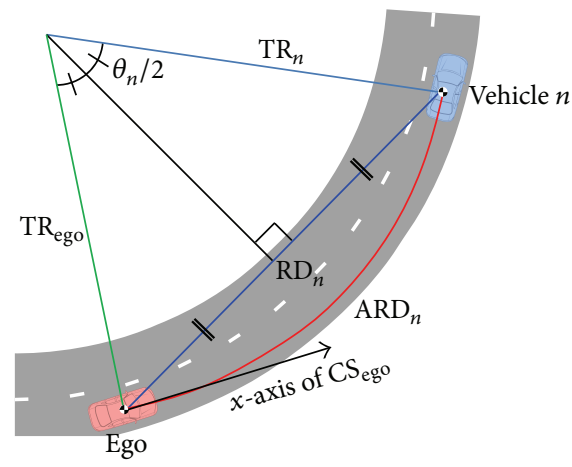

FIgURE 5: Arc Relative Distance (ARD) on a curved road.

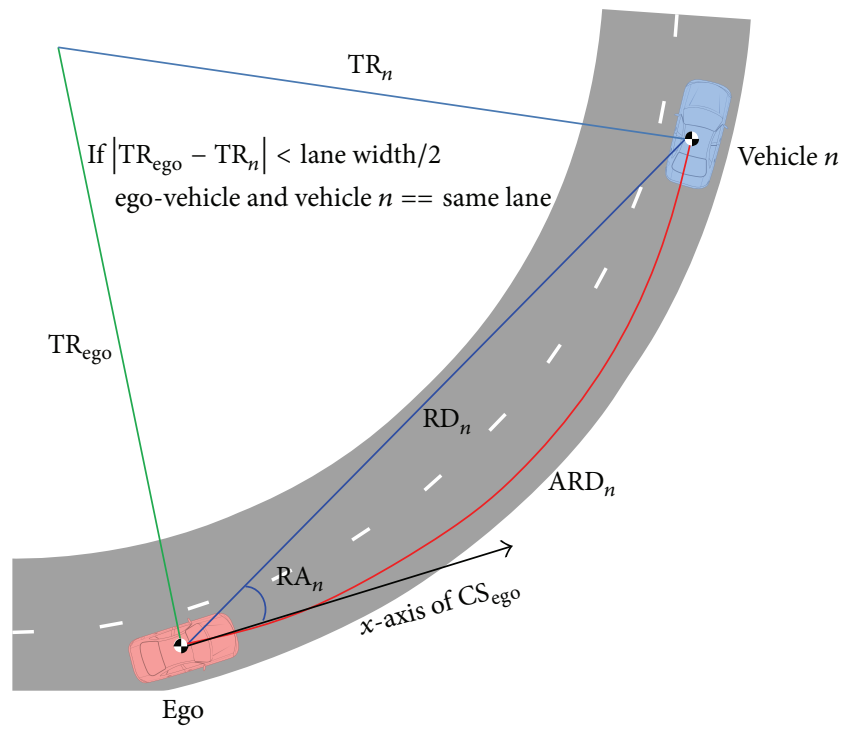

FIGURE 6: Estimation of radius of rotation and decision of same lane with vehicle $n$.

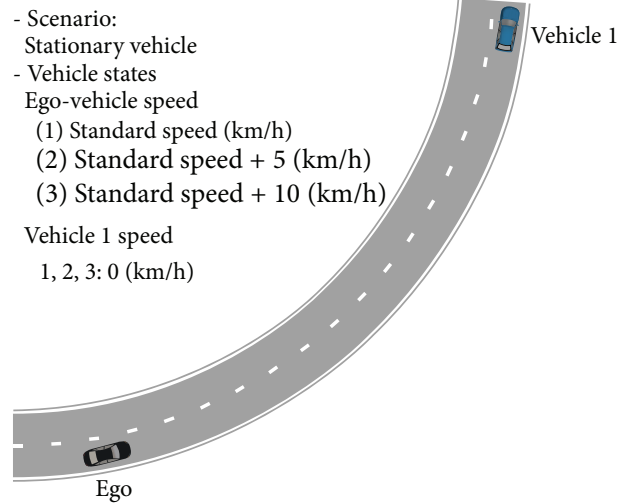

FIGURE 7: Simulation scenario.

TABLE 1: Standard speed of vehicle according to the radius of curvature of a curved road.

\begin{tabular}{lcccc}
\hline Scenario number & 1 & 2 & 3 & 4 \\
\hline Radius of curvature $[\mathrm{m}]$ & 15 & 30 & 60 & 90 \\
\hline Standard speed $[\mathrm{km} / \mathrm{h}]$ & 20 & 30 & 40 & 50 \\
\hline Superelevation $[\%]$ & 6 & 6 & 6 & 6 \\
\hline Initial relative distance $[\mathrm{m}]$ & 42 & 85 & 145 & 175 \\
\hline
\end{tabular}

TABLE 2: Definition of warning level according to changes in $\mathrm{TTC}_{x}$.

\begin{tabular}{|c|c|c|c|}
\hline \multicolumn{2}{|c|}{ Degree of risk } & \multicolumn{2}{|c|}{ Low $\longleftrightarrow$ high } \\
\hline $\mathrm{TTC}_{x}[\mathrm{sec}]$ & $\leq 2.7$ & $\leq 1.7$ & $\leq 0.8$ \\
\hline Warning level & 1 & 2 & 3 \\
\hline
\end{tabular}

difference between the time of collision and the simulated time, because the vehicle is being driven at a constant speed in the scenario. Figure 8 shows the margin of error used when comparing the $\mathrm{TTC}_{x \text {,ideal }}$ (which is the ideal longitudinal 


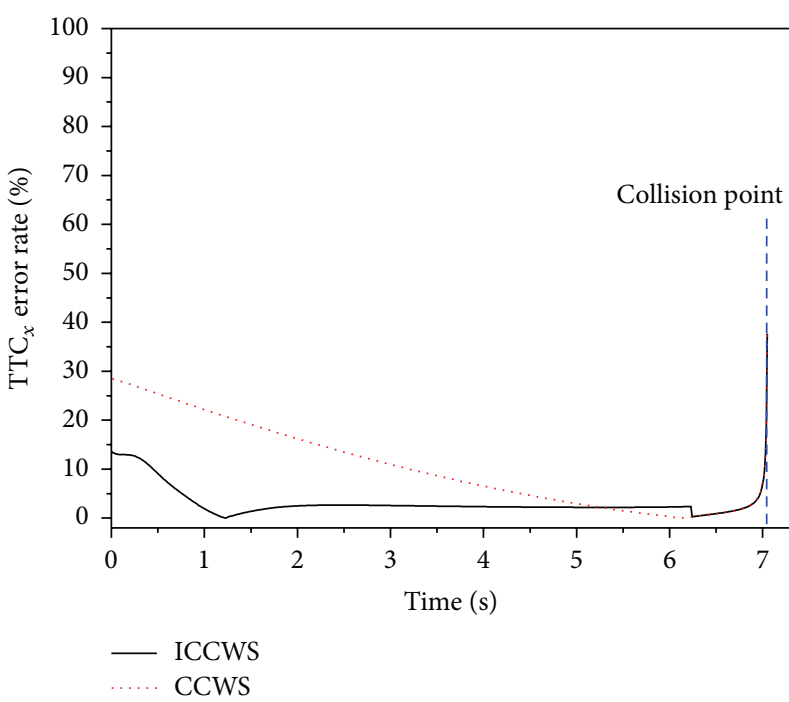

(a)

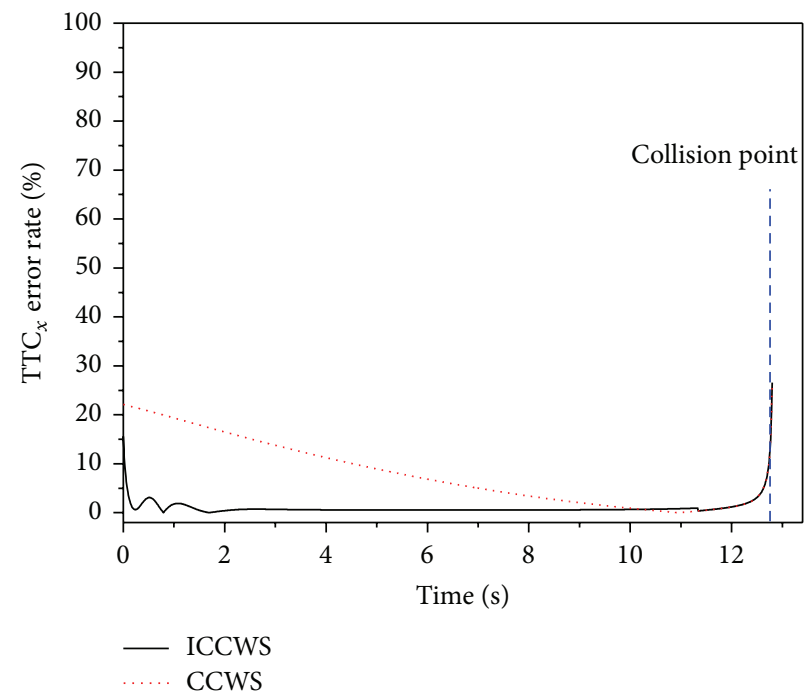

(c)

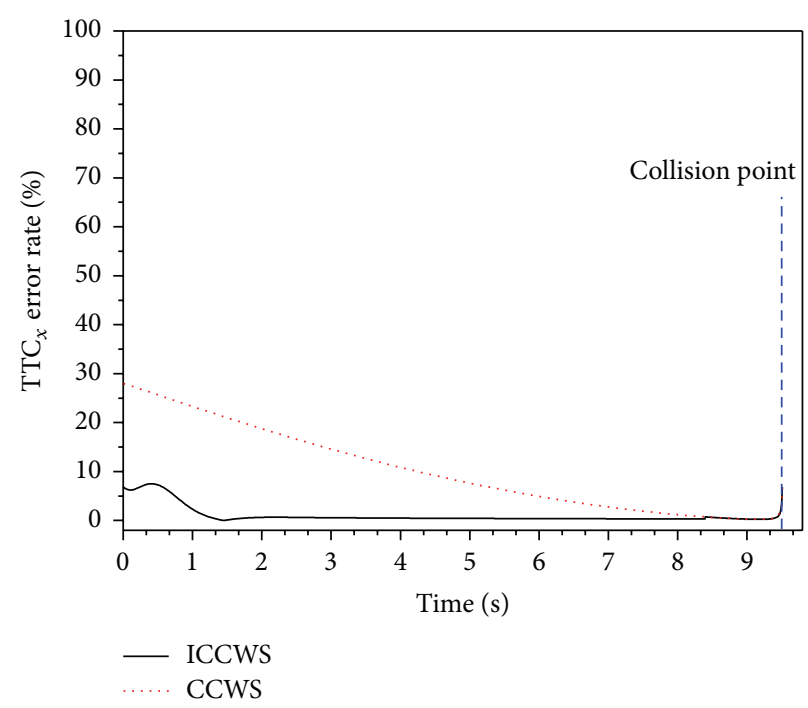

(b)

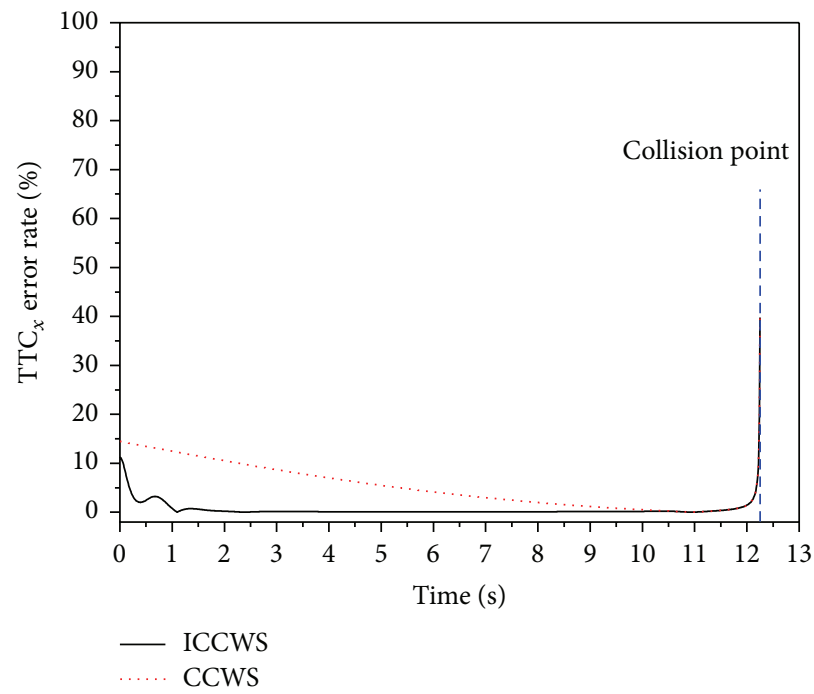

(d)

FIgURE 8: Margin of error of collision risk index of ICCWS and CCWS: (a) Scenario 1; (b) Scenario 2; (c) Scenario 3; and (d) Scenario 4.

collision risk index) with each $\mathrm{TTC}_{x}$, calculated using the proposed ICCWS and the existing CCWS, after simulation of identical scenarios (Scenario 1, 2, or 3). The results of $\mathrm{TTC}_{x, \mathrm{ICCWS}}$, which is the longitudinal collision risk index of the proposed ICCWS corresponding to each scenario, indicate the following approximate average margins of error: for Scenario $1($ Figure $8(\mathrm{a}))=3.18 \%$, Scenario $2($ Figure $8(\mathrm{~b}))=$ $1.06 \%$, Scenario 3 (Figure $8(\mathrm{c}))=1.15 \%$, and Scenario 4 (Figure $8(d))=0.59 \%$. These results confirm that the calculation of collision risk is relatively accurate. The reason for a $\mathrm{TTC}_{x, \text { ICCWs }}$ error in the initial simulation is related to an error in the vehicle's TR owing to the rapidly changing value of the yaw rate when the ego-vehicle enters the curved road. However, the margin of error declines as the ego-vehicle begins a stable turn (after approximately $1.3 \mathrm{~s}$ ), and thus the correct estimation of the collision risk can be confirmed.
The CCWS showed the following approximate average margins of error of the CCWS corresponding to each scenario: Scenario 1 (Figure $8(a))=10.67 \%$, Scenario 2 $($ Figure $8(b))=10.32 \%$, Scenario $3($ Figure $8(c))=8.17 \%$, and Scenario 4 (Figure $8(\mathrm{~d}))=5.23 \%$. This confirms a difference of approximately $7.10 \%$ compared to the proposed ICCWS. The reason for this difference is that the existing CCWS calculates the $\mathrm{TTC}_{x, \mathrm{CCWS}}$, which is the longitudinal collision risk index, without considering the curvature of the road. Table 3 presents the results of a comparative analysis simulation using the application of the ICCWS, CCWS, and FCWS (based on vehicle-mounted sensors) on the scenarios defined in Table 1. The simulation results listed in Table 3 show the margins of error, presented according to the scenarios. According to the results, the FCWS that uses a vehicle on-board sensor has a limited measurable range on curved roads. In other words, 
TABLE 3: Margin of error, ICCWS, CCWS, and FCWS.

\begin{tabular}{|c|c|c|c|}
\hline & $\begin{array}{c}\text { Standard } \\
\text { speed }[\mathrm{km} / \mathrm{h}]\end{array}$ & $\begin{array}{c}\text { Collision } \\
\text { warning system }\end{array}$ & $\begin{array}{c}\text { Margin of error } \\
{[\%]}\end{array}$ \\
\hline \multirow{9}{*}{ Scenario 1} & \multirow{3}{*}{20} & ICCWS & 3.18 \\
\hline & & CCWS & 10.67 \\
\hline & & FCWS & 83.40 \\
\hline & \multirow{3}{*}{25} & ICCWS & 2.40 \\
\hline & & CCWS & 10.00 \\
\hline & & FCWS & 81.73 \\
\hline & \multirow{3}{*}{30} & ICCWS & 2.96 \\
\hline & & CCWS & 10.48 \\
\hline & & FCWS & 81.76 \\
\hline \multirow{9}{*}{ Scenario 2} & \multirow{3}{*}{30} & ICCWS & 1.06 \\
\hline & & CCWS & 10.32 \\
\hline & & FCWS & 78.20 \\
\hline & \multirow{3}{*}{35} & ICCWS & 0.78 \\
\hline & & CCWS & 10.17 \\
\hline & & FCWS & 77.92 \\
\hline & \multirow{3}{*}{40} & ICCWS & 2.65 \\
\hline & & CCWS & 12.38 \\
\hline & & FCWS & 80.07 \\
\hline \multirow{9}{*}{ Scenario 3} & \multirow{3}{*}{40} & ICCWS & 1.15 \\
\hline & & CCWS & 8.17 \\
\hline & & FCWS & 79.30 \\
\hline & \multirow{3}{*}{45} & ICCWS & 1.94 \\
\hline & & CCWS & 8.66 \\
\hline & & FCWS & 79.88 \\
\hline & \multirow{3}{*}{50} & ICCWS & 2.20 \\
\hline & & CCWS & 8.68 \\
\hline & & FCWS & 79.80 \\
\hline \multirow{9}{*}{ Scenario 4} & \multirow{3}{*}{50} & ICCWS & 0.59 \\
\hline & & CCWS & 5.23 \\
\hline & & FCWS & 82.58 \\
\hline & \multirow{3}{*}{55} & ICCWS & 1.33 \\
\hline & & CCWS & 5.70 \\
\hline & & FCW & 82.96 \\
\hline & \multirow{3}{*}{60} & ICCWS & 1.11 \\
\hline & & CCWS & 5.53 \\
\hline & & FCWS & 83.19 \\
\hline
\end{tabular}

it exhibits a margin of error that is greater than $79 \%$ in all of the scenarios. On the other hand, the ICCWS proposed in this study exhibits a margin of error that is, at most, less than $3 \%$ in all of the scenarios and is similar to the actual performance of following a preceding vehicle. Moreover, compared to CCWS, which does not consider the curvatures of roads, ICCWS reduces the margin of error to a maximum of approximately $7 \%$ for roads with large curvatures. By improving the performance of following a preceding vehicle, safe driving can be ensured because the driver can recognize accurate risk-warning signals on curved roads.

\section{Conclusion}

This research proposes an ICCWS that considers a small curvature radius on curved roads. ARD, which is the actual relative distance between the ego-vehicle and the preceding vehicle on the curved road, was calculated by utilizing the turning radius of the ego-vehicle and data obtained from the vehicle surroundings monitoring system. As per the results, we reduced a maximum of approximately $7 \%$ margin of error compared to CCWS and $82 \%$ compared to FCWS. With the improvement in following a preceding vehicle using ICCWS proposed in this study, more accurate risk-warning signals were provided to drivers on curved roads, and, thus, driver resistance to the system was minimized.

In future research, the application of the proposed ICCWS to the primary collision evasion system, autonomous emergency braking (AEB), is expected, in addition to studies on the implementation of the ICCWS on various roads and within multivehicle environments.

In conclusion, this study proposed an ICCWS to overcome the problems of the conventional FCWS and CCWS, without adding devices to the vehicle.

\section{Conflict of Interests}

The authors declare that there is no conflict of interests regarding the publication of this paper.

\section{Acknowledgment}

This work was supported by the 2013 Research Fund of University of Ulsan.

\section{References}

[1] NHTSA, "The impact of driver inattention on near-crash/crash risk: an analysis using the 100-car naturalistic driving study data," NHTSA Report, National Highway Traffic Safety Administration, 2006.

[2] K. Goswami, G. Hong, and B. Kim, "A novel mesh-based moving object detection technique in video sequence," Journal of Convergence, vol. 4, no. 3, pp. 20-24, 2013.

[3] D. Caveney and W. B. Dunbar, "Cooperative driving: beyond $\mathrm{V} 2 \mathrm{~V}$ as an ADAS sensor," in Proceedings of the IEEE Intelligent Vehicles Symposium (IV '12), pp. 529-534, June 2012.

[4] Z. C. Taysi and A. G. Yavuz, "ETSI compliant GeoNetworking protocol layer implementation for IVC simulations," Humancentric Computing and Information Sciences, vol. 3, article 4, 2013.

[5] R. Hussain and H. Oh, "Cooperation-aware VANET clouds: providing secure cloud services to vehicular ad hoc networks," Journal of Information Processing Systems, vol. 10, no. 1, pp. 103118,2014

[6] ISO, "Transport information and control systems forward vehicle collision warning systems performance requirements and test procedures," ISO 15623, International Organization for Standardization, London, UK, 2013. 
[7] K. D. Kusano and H. C. Gabler, "Safety benefits of forward collision warning, brake assist, and autonomous braking systems in rear-end collisions," IEEE Transactions on Intelligent Transportation Systems, vol. 13, no. 4, pp. 1546-1555, 2012.

[8] A. Sebastian, M. Tang, Y. Feng, and M. Looi, "Multi-vehicles interaction graph model for cooperative collision warning system," in Proceedings of the IEEE Intelligent Vehicles Symposium, pp. 929-934, IEEE, Xian, China, June 2009.

[9] H. Cho, G.-E. Kim, and B.-W. Kim, "Usability analysis of collision avoidance system in vehicle-to-vehicle communication environment," Journal of Applied Mathematics, vol. 2014, Article ID 951214, 10 pages, 2014.

[10] E. Ryu, S. Jo, J. Kwon, T. Hong, and K. Park, "A study on the development of risk situation awareness algorithm and performance verification with PreScan," in Proceedings of the Conference for Korea Society of Automotive Engineers, pp. 605-613, May 2013. 


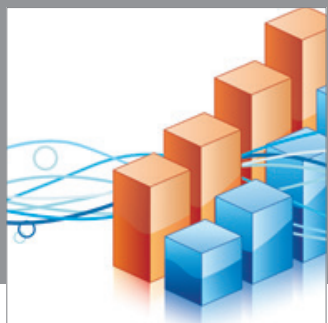

Advances in

Operations Research

mansans

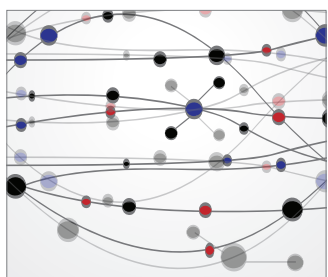

The Scientific World Journal
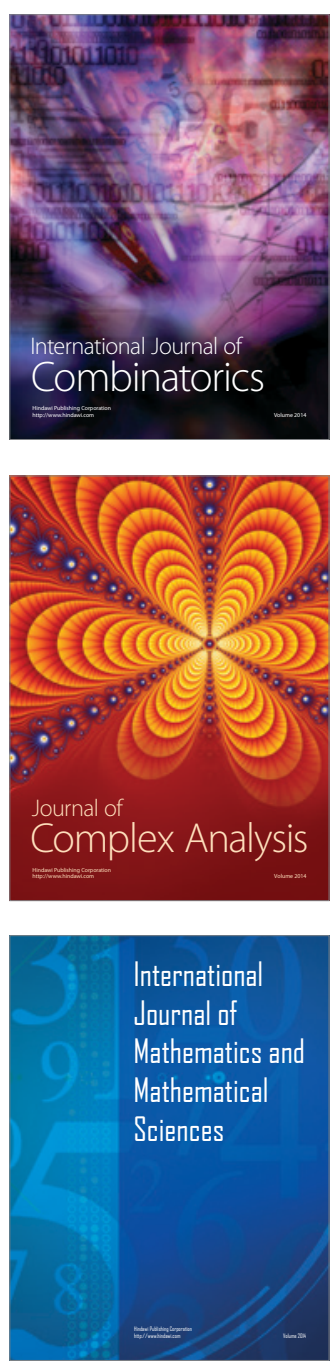
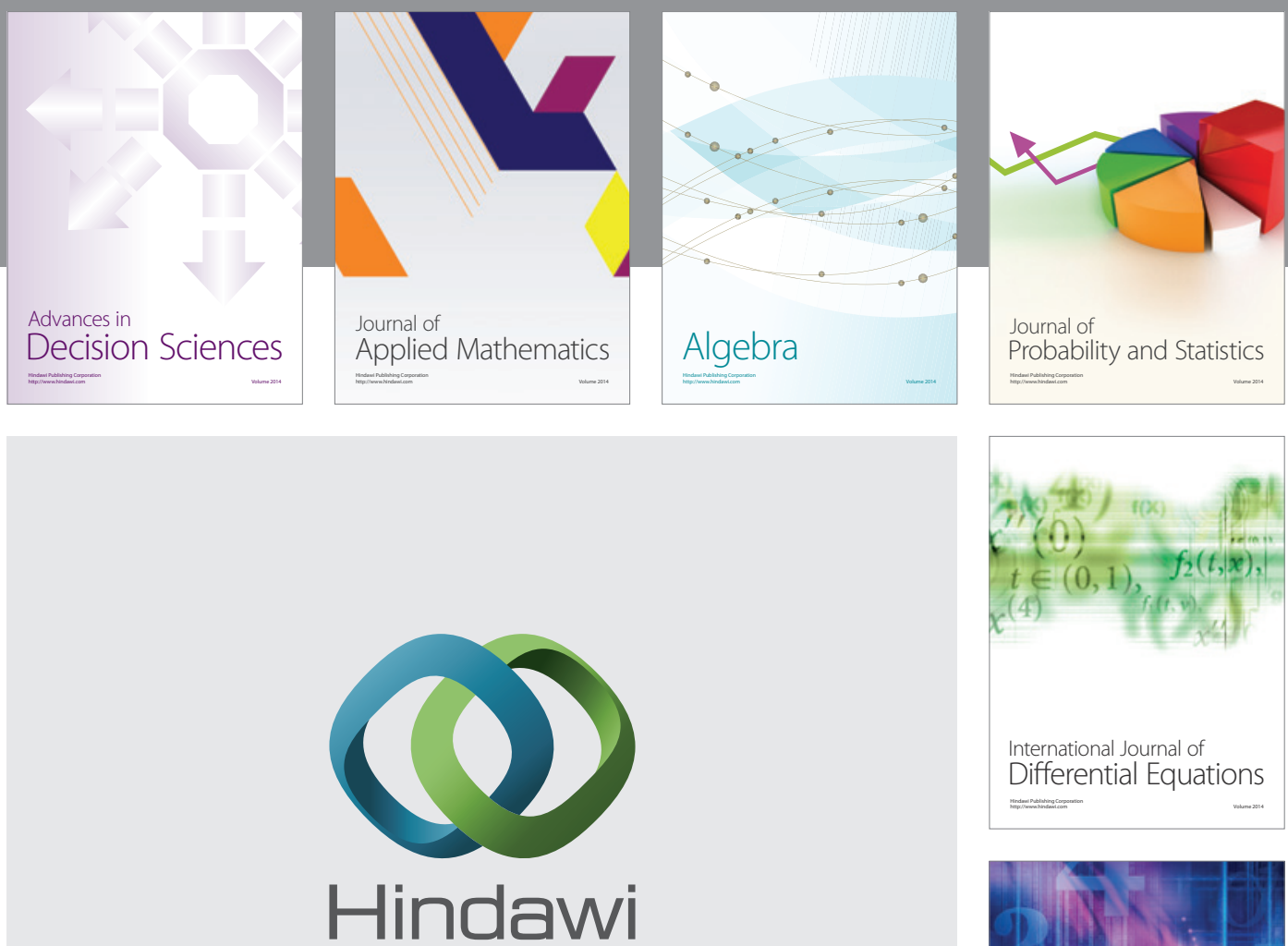

Submit your manuscripts at http://www.hindawi.com
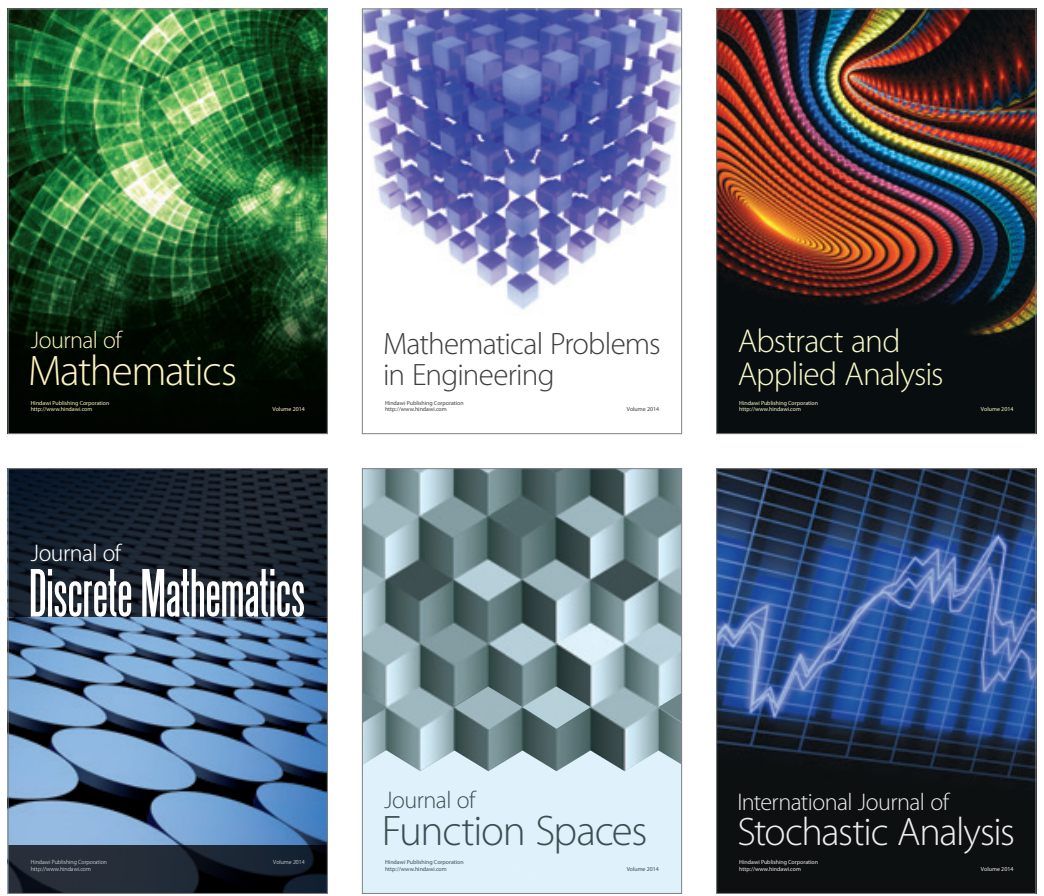

Journal of

Function Spaces

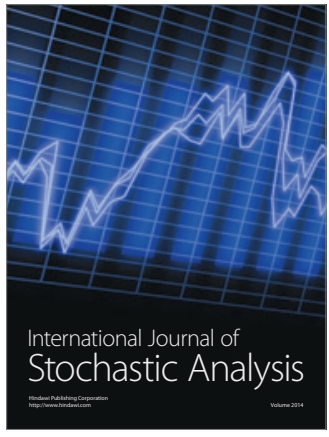

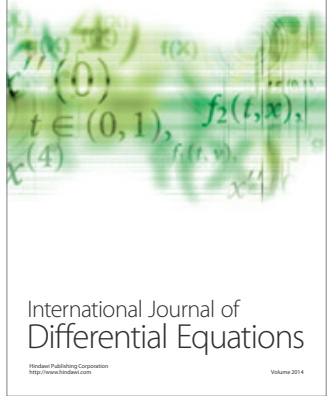
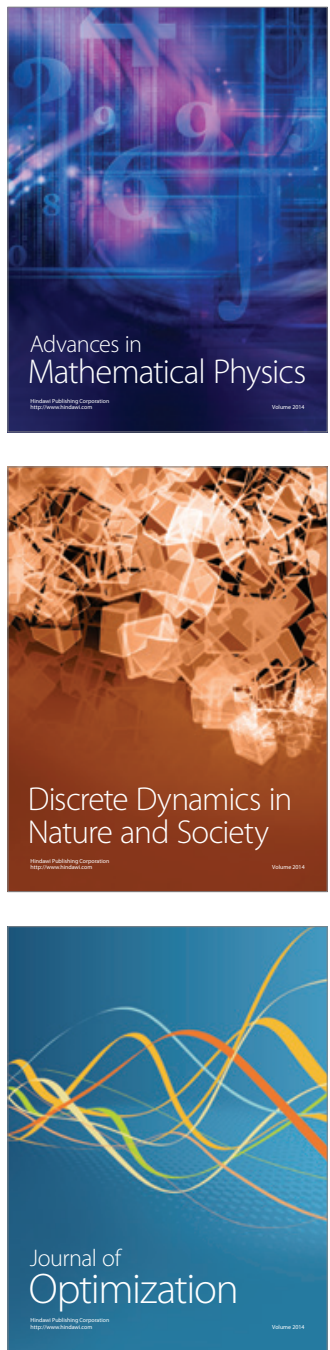